\title{
Reconstruction of Two-Dimensional to Three- Dimensional Ultrasound Images Through Volume Rendering Method Using ImageJ
}

\author{
Ayu Fitri Amalia ${ }^{1}$, Arif Bintoro ${ }^{2}$, Bayu R Setiadi ${ }^{3}$, Gede Bayu Suparta ${ }^{4}$ \\ \{ayufitriamalia@ustjogja.ac.id ${ }^{1}$, arif.bintoro@ustjogja.ac.id ${ }^{2}$, bayursetiadi@gmail.com ${ }^{3}$, \\ gbsuparta@ugm.ac.id\} \\ Physics Education Department ${ }^{1}$, Mechanical Engineering Education Department ${ }^{23}$, Universitas \\ Sarjanawiyata Tamansiswa \\ Yogyakarta, Indonesia ${ }^{123}$ \\ Physics Science Department ${ }^{4}$ Universitas Gadjah Mada \\ Yogyakarta, Indonesia ${ }^{4}$
}

\begin{abstract}
Two approaches of 3D reconstruction process can be used which is either 3D surface model or voxel-based volume. The rendering technique determines the ability to visualize information in the 3D image. Surface-based viewing techniques, multiplane viewing techniques and volume-based rendering techniques are three basic types being used to visualize information in the $3 \mathrm{D}$ image. This research aimed to analyze the quality of the images obtained through a simple method of $2 \mathrm{D}$ visualization and reconstruction of 2D into 3D images by using image processing applications through 2D multiple image capture. Based on the results of his study, in the volume rendering technique, the view volume displayed through the coordinates $\mathrm{xy}, \mathrm{yz}$, and $\mathrm{xz}$ has not made it easy to observe the physiological structure of the uterus object in 3D. In xy coordinates, the uterus area can be seen more clearly and can be distinguished from the area of other organs around it. (Abstract)
\end{abstract}

Keywords: component, formatting, style, styling, insert (key words)

\section{Introduction}

Today's technological developments make computer systems capable high computing to improve data processing into information[1]. Technology research and development in the biomedical field is developing with fast. A lot of research is done to create new discoveries to solve various problems that continue to arise along with technological progress. As technology develops in the field of Information and Computer Technology, three-dimensional (3D) technology also develops rapidly. The need to visualize 3D objects is widely used in animation and graphics applications, architecture, education, medical, and cultural recognition, as well as virtual reality.

Medical imaging is the imaging technique of the human body for clinical purposes especially for analyzing some anatomy related to abnormalities. Ultrasound (US), CT, and 
MRI are some of the commonly used imaging techniques[2]. Almost the entire range of internal organs in the abdomen including the genitourinary system which consists of kidneys, urinary bladder, urethra and reproductive system of male and female images were obtained through the diagnostic US. Due to dynamically display two-dimensional (2D) images of the Region of Interest in real-time, conventional 2D US has been widely used[3], [4]. The diagnostic accuracy through 2D US much uncertain as it heavily depends on the experience and knowledge of clinicians.

However, conventional 2D only a two-dimensional frame is produced at a given time. So that, 2D US imaging has limitations in quantifying the volume of structures of interest in the body. Volume quantification is important in assessing the progression of a disease and tracking the progression of response to treatment. An application that can transform a 2D image data collection into a $3 \mathrm{D}$ visualization that is close to its original form is necessary so that users can be helped to interpret the information. All recent medical 3D image reconstruction techniques create 3D images from sets of 2D slices (Fig.1), which can be recorded by various equipments such as CT, MRI, ultrasound etc[5].

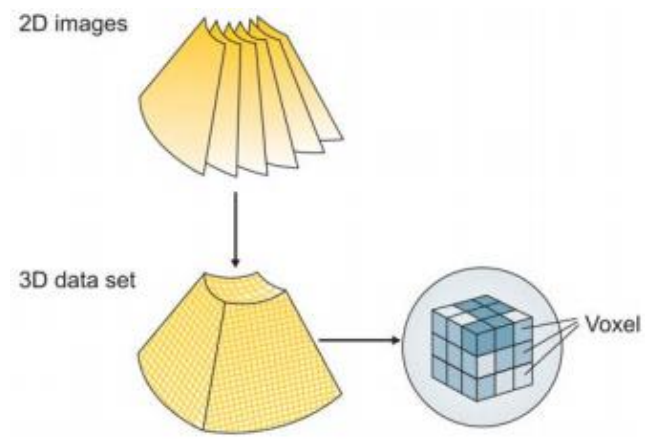

Fig.1. Construction of a 3D data set[6]

Two approaches of 3D reconstruction process can be used which is either 3D surface model or voxel-based volume. The rendering technique determines the ability to visualize information in the 3D image. Surface (SR), multiplanar (MPR), and volume (VR) rendering techniques are three basic types being used to visualize information in the $3 \mathrm{D}$ image[7]. MPR is appropriate for low configuration computers. It doesn't require too many calculations. With axial slices, we can use it to reslice according to different directions such as coronal, sagittal or diverse. SR technique is used when we want to see the surfaces of a structure separately from near structure, e.g. skull from slices of head, blood vessel system from slices of body etc. SR technique is often used for high contrast data. VR technique is used to visualize the entire volume transparence of the object that helps us to see comprehensively an entire compact structure of the object. Images will be performed by projecting rays through volume data. But, it is enormous amount of calculations, which requires strong configuration computers. This technique is appropriate for low contrast data[5].

Image J is scientific software that allows us to explore our data and to control exactly what happens to it. It was developed at the National Institutes of Health by Wayne Rasband. The ' $\mathrm{J}$ ' stands for Java: the programming language in which it is written. It can be downloaded for free from http://imagej.net, and its source code is in the public domain, making it particularly easy to modify and distribute[8]. 
An image must be presented numerically with discrete values. So that, it is processed with a digital computer. A digital image can be represented by a two-dimensional matrix $\mathrm{f}(\mathrm{x}, \mathrm{y})$ consisting of $\mathrm{M}$ columns and $\mathrm{N}$ lines, where intersections between columns and rows are called pixels. The spread of the pixel intensity of an image or certain part of the image is represented in a graphic form called a histogram. The histogram shows the number of grey level (0-255) that provides a description of the brightness or contrast of an image[9]. The Histogram of a digital image is a discrete function. The intensity levels are in the range $[0, \mathrm{~L}-$ 1], as in Equation.1[10].

$$
h\left(r_{k}\right)=n_{k}
$$

In this research, the means to produce 3D US images were obtained by time-lapsed 2D US capture techniques, ie each frame will be captured at a speed lower than the speed when the frame is played. So that, the movement of objects from the initial position of the transducer until the final position of the transducer can be recorded. Then the obtained images are used to reconstruct using ImageJ applications to form 3D US images and analyze them.

\section{Method}

The management of the research was carried out following a flowchart such as Figure 2. The US imaging process was carried out by arranging US imaging equipment such as Figure 3.

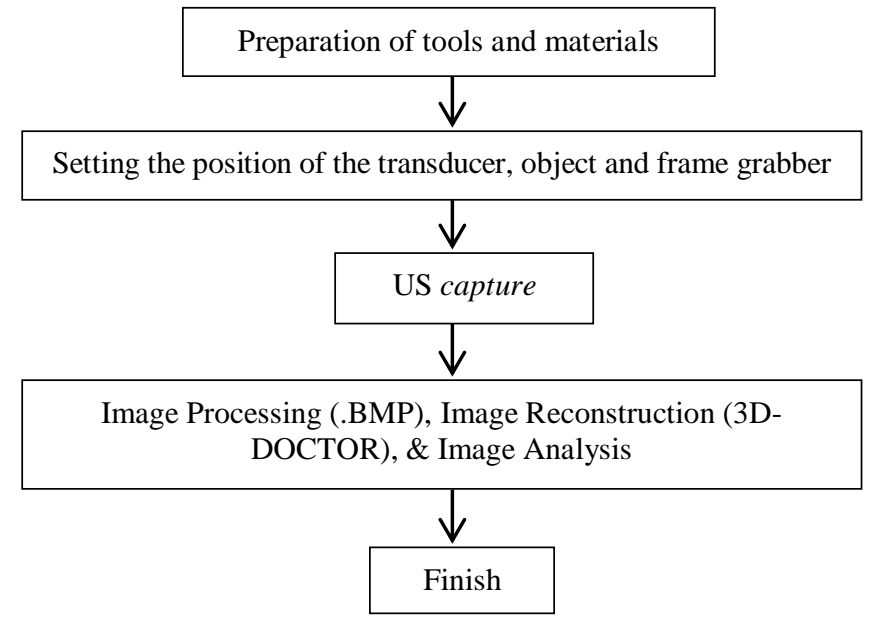

Fig.2. Research flow chart 


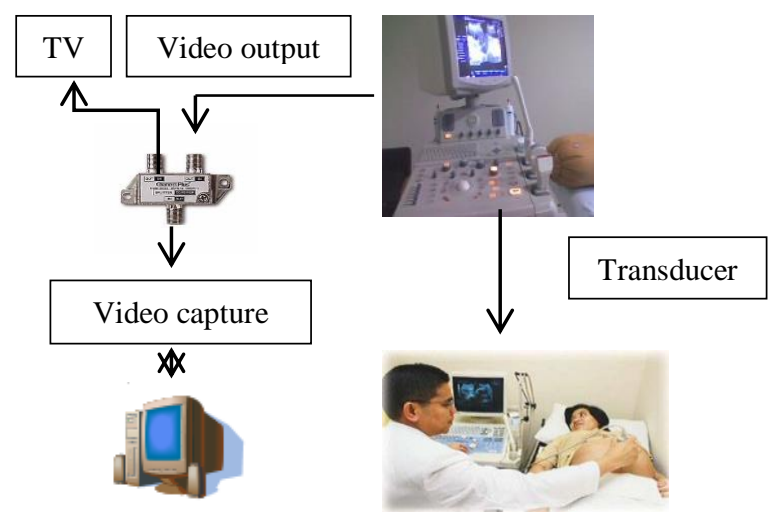

Fig.3. US imaging equipment

\section{Result and Discussion}

\subsection{US Image Processing}

The process of ultrasound image processing and reconstruction is carried out from several 2D US slices shown. Observations were made using time-lapsed image recording techniques with a 50 milliseconds inter-image recording interval for 5 seconds which produced 100 2D ultrasound images.

In the use of 3D-DOCTOR applications, it began by uploading a set of 2D images and will be visualized into 3D images. Then the cropping process was done by first determining the 2D image area which is done by selecting the Region of Interest (ROI) command. The next step was to determine the area of the image to be cropped, represented by a $2 \mathrm{D}$ image by drawing a polygon line from one point to another so as to border the cropped area. The display of Crop Image by ROI can be seen in Figure 4. A set of 2D image data that has been cropped, has automatically been saved by 3D-DOCTOR in the TIF format and shown in Figure 5. A set of images was stored in a stacked state according to the order.

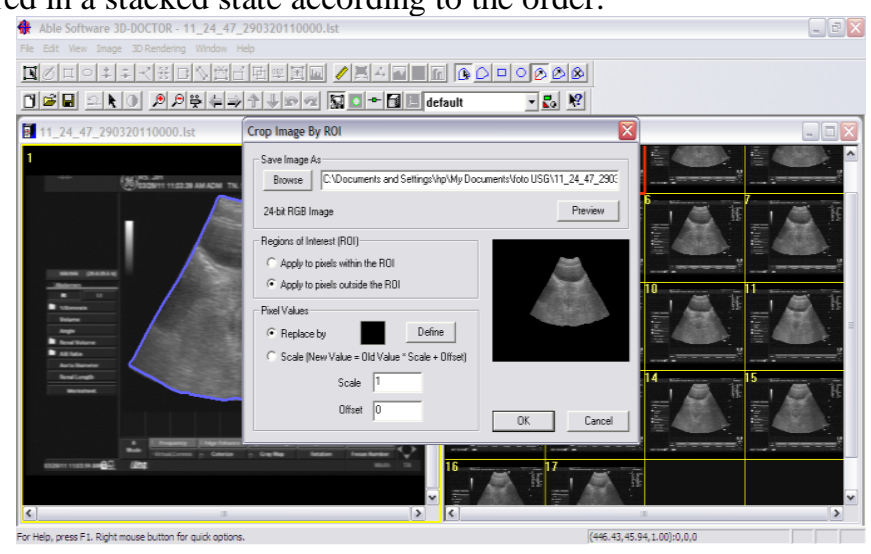

Fig.4. Crop 3D Image by ROI 


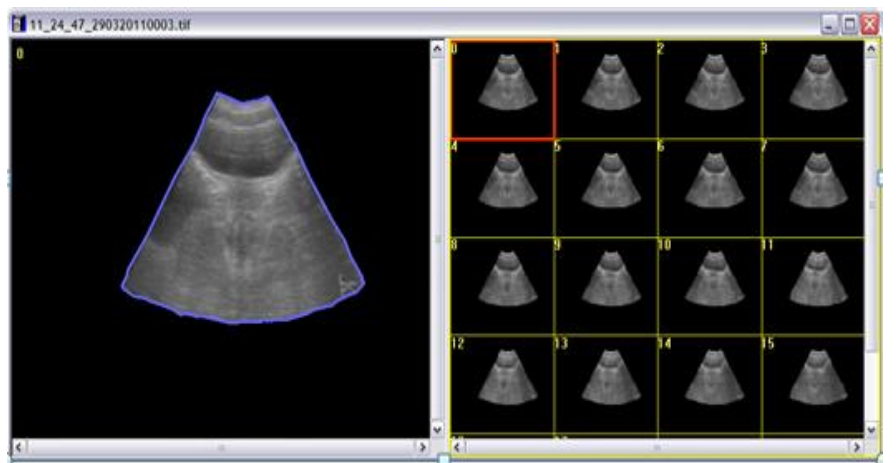

Fig.5. Cropping image processing results through 3D-DOCTOR application

After the cropping process, image normalization was then carried out. The ImageJ application can do the normalization process for a $2 \mathrm{D}$ image data set. Furthermore, the reconstruction stage was carried out through volume rendering. At this stage, a set of $2 \mathrm{D}$ US images will be reconstructed into 3D images. Before being reconstructed, the cropping image and stack were image processing in the form of enhancing contrast on the ImageJ application.

2D US image contrast that has been cropped and stacked was shown through a histogram image via the ImageJ application as Figure 6. From this histogram, this 2D US image has a narrow curve histogram. The distribution of bright and dark intensity was uneven.

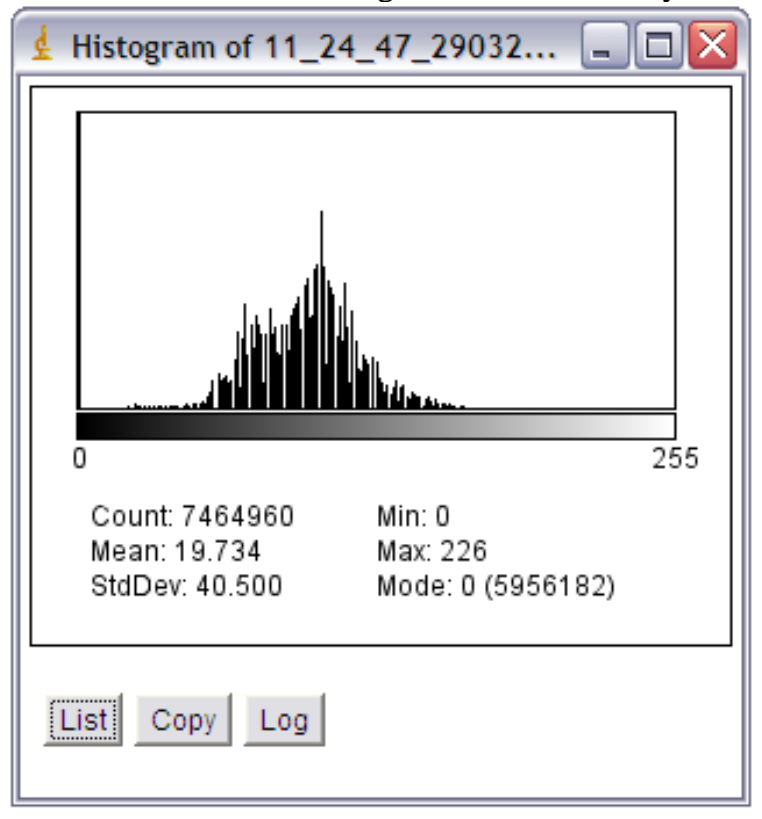

Fig.6. 2D US image histogram after cropping and stacking

In the histogram, the darkest point has an intensity of 0 and the brightest one has an intensity of 226. This meant that the brightest point did not reach the bright white colour at the intensity of 255. So, it was necessary to enhance contrast. 2D US images that had been cropped and 
stacked were reopened through the ImageJ application. The 2D US stacked image that had been enhanced contrast was shown in Figure 7.

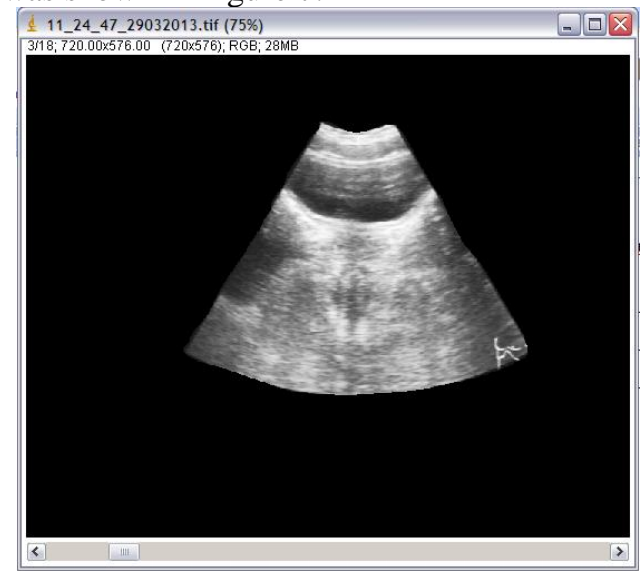

Fig.7. 2D US stack image after contrast enhancement

After enhancing the contrast, the differences between the areas of the reproductive organs of women have been clearer. A 2D US image histogram that has been contrast-enhanced can be seen in Figure 8. From the histogram, the 2D ultrasound image experienced histogram equalization. Intensity values in the image have been changed so that their distribution was uniform. Each gray level had the same relative number of pixels so that the histogram has been evenly distributed throughout the gray level. Here, this 2D ultrasound image had a gray level from 0 to 255 .

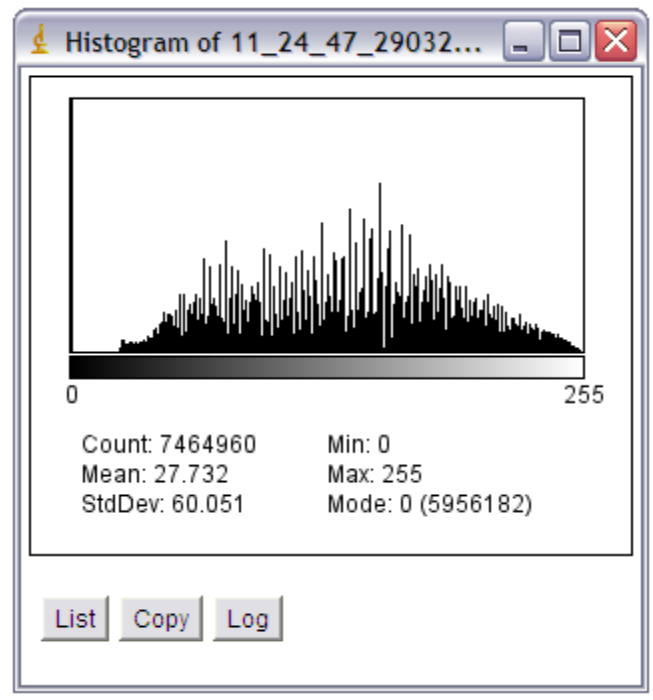

Fig.8. 2D US image histogram after contrast enhancement 


\subsection{D US Visualization}

In the volume rendering model, a set of $2 \mathrm{D}$ uterus US images will be reconstructed into $3 \mathrm{D}$ uterus US images. A set of 2D images is stacked according to the order after image processing has been done previously. These piles of images make 3D US volume images from 2D US images. In the ImageJ application, this stage is done through 3D Volume Viewer. The result is a $3 \mathrm{D}$ volume image display that can be displayed via $\mathrm{xy}, \mathrm{yz}$, and $\mathrm{xz}$ coordinates.

The volume view displayed through $\mathrm{xy}, \mathrm{yz}$, and $\mathrm{xz}$ coordinates has not made it easy to observe the physiological structure of the 3D uterus. Even though the xy coordinates, the uterus area can be seen more clearly and distinguished from other areas of the organ around it (Figure 9). It was shown cross-sections through that point from each direction.

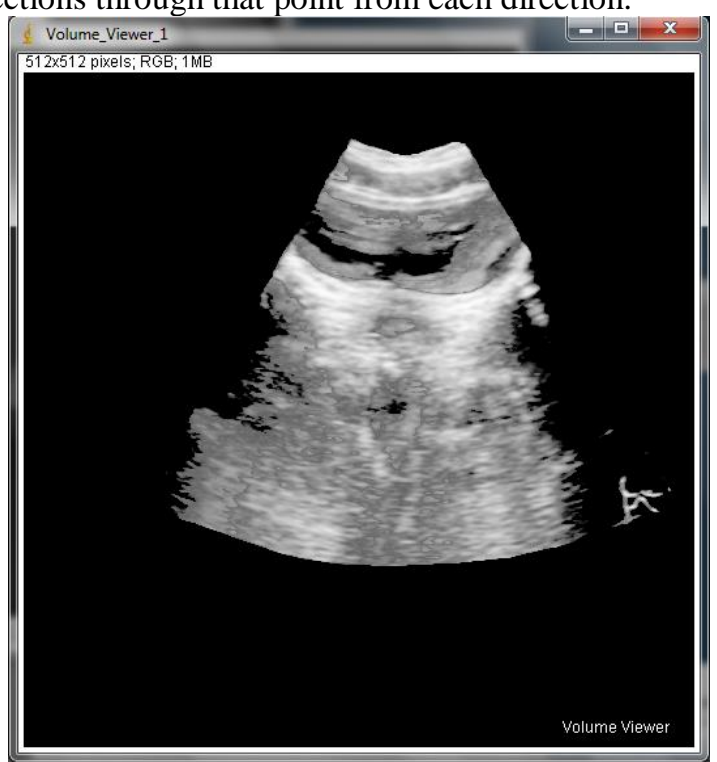

Fig.9. View volume of 3D US images of uterus via $x y$ coordinates

When viewed from $y z$ and $x z$ coordinates, only obtained image slices from a set of 2D ultrasound images. The 3D uterus image seen in the yz (Figure 10) and xz (Figure 11) coordinates did not indicate the depth or thickness of the uterus. However, if the image of the $3 \mathrm{D}$ uterus in xy coordinates seen visually, it will look like the image of the surface of the 3D uterus that was the image of the uterus as it surfaces from the surrounding area. This is because the image of the 3D uterus has a brighter intensity than the image outside the uterine area.

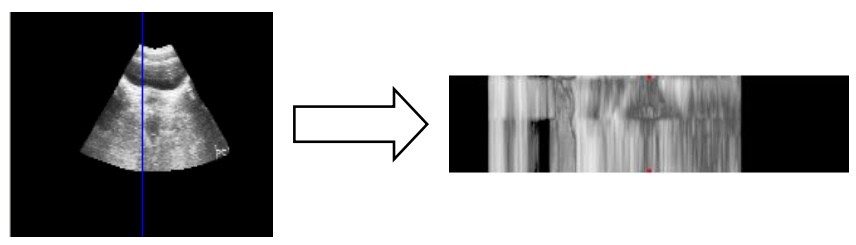

Fig.10. View volume of 3D US images of uterus via $y z$ coordinates 


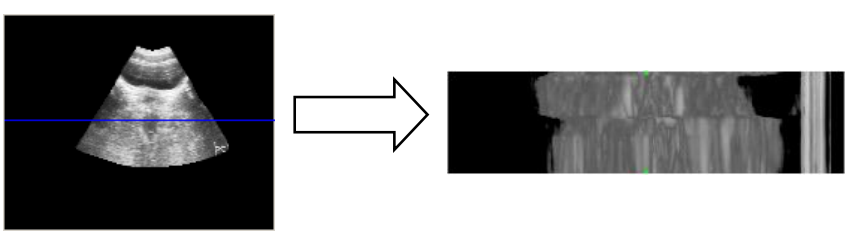

Fig.11. View volume of 3D US images of uterus via $x y$ coordinates

\section{Conclusions}

Based on the results of his study, in the volume rendering technique, the view volume displayed through the coordinates $x y, y z$, and $x z$ has not made it easy to observe the physiological structure of the uterus object in 3D. In xy coordinates, the uterus area can be seen more clearly and can be distinguished from the area of other organs around it.

\section{Acknowledgment}

We are grateful to teamwork in Grup Riset Fisika Citra (GRFC) UGM for numerous discussions on the subject of this work. This research is based upon work supported by GRFC \& LP3M UST.

\section{References}

[1] A. F. Amalia and H. Saputro, "Analisis deteksi iris mata menggunakan metode deteksi tepi sobel," Sci. TECH J. Ilm. Ilmu Pengetah. dan Teknol., vol. 4, no. 1, pp. 41-48, 2018.

[2] M. Hafizah, T. A. N. Kok, and E. K. O. Supriyanto, "Development of 3D Image Reconstruction Based on Untracked 2D Fetal Phantom Ultrasound Images using VTK," vol. 6, no. 4, pp. 145-154, 2010

[3] Q. Huang, F. Yang, L. Liu, and X. Li, "Automatic Segmentation of Breast Lesions for Interaction in Ultrasonic Computer-Aided Diagnosis,” Inf. Sci. (Ny)., 2014.

[4] Q. Huang, Y. Luo, and Q. Zhang, "Breast ultrasound image segmentation : a survey," 2017.

[5] V. Cong and H. Q. Linh, "3D MEDICAL IMAGE RECONSTRUCTION," pp. 1-5.

[6] K. Baba, "Development of 3D Ultrasound," vol. m, no. September, pp. 205-215, 2010.

[7] S. Ko, S. S. Hong, J. Hwang, and H. Kim, "Application of Three-Dimensional Volumetric Ultrasonography in Patients with Bladder Cancer and Its Mimickers: A Pictorial Essay 방광암과 유사 질환의 감별에 있어 3차원 초음파의 적용 : 임상 화보," vol. d, no. 5, pp. 346-353, 2017.

[8] P. Bankhead, Analyzing fluorescence microscopy images with ImageJ, no. May. Belfast, 2014.

[9] A. F. Amalia, W. Budhi, U. N. Prabowo, and G. Bayu, "multiple image capturing The Image Quality Analysis of Neutron Digital Radiography Through The Variation of Multiple Image Capturing,” vol. 020140, 2018.

[10] K. Vij and Y. Singh, "Enhancement of images using histogram processing techniques," Int. J. Comput. 
Technol. Appl., vol. 2, no. 2, pp. 309-313, 2009. 